\title{
Scientific Validation on the Effect of Siddha Sashtric Formulations in Chemotherapy Induced Nausea and Vomiting - A Review
}

\author{
Vigneshwari $\mathbf{S}^{1}$, Antony Duraichi $\mathbf{R}^{2}$ \\ ${ }^{1}$ PG Scholar, Department of Gunapadam, ${ }^{2}$ Lecturer, Department of Gunapadam, \\ Government Siddha Medical College, Palayamkottai, Tirunelveli, Tamil Nadu, India.
}

Corresponding Author: Vigneshwari S

\begin{abstract}
Siddha system is one of the major Indian systems of medicine. Siddha sashtric formulations are used for the treatment and management of non communicable diseases like stroke, cancer, diabetes, chronic lung diseases etc. Of these, cancer is the second leading cause of death globally. CINV is a significant burden for patients undergoing anticancer therapy. Nausea and vomiting are rated as two of the most distressing symptoms by chemotherapy patients and have been shown to significantly and adversely affect quality of life and physical function during treatment (Carelle et al., 2002). Many siddha sashtric formulations are available for nausea and vomiting. All of the available information about Siddha anti emetic Formulations, their ingredients and chemical constituents were collected from the rich collection of ancient Siddha books held in the library, Government Siddha Medical College, Palayamkottai, Tirunelveli. Electronic searches also made. They also have the therapeutic actions like anticancer, antioxidant, anti inflammatory, chemo preventive, gastro protective etc. This review explains the scientific evidences for the effect of Siddha Sashric formulations in the treatment and management of chemotherapy induced nausea and vomiting. This documentation provides the baseline information about the therapeutic importance of Siddha medicines in chemotherapy induced nausea and vomiting and it will be useful for further clinical trials.
\end{abstract}

Key Words: Siddha medicine, cancer, chemotherapy, CINV, nausea and vomiting.

\section{INTRODUCTION}

Globally about one in six deaths is due to cancer (who). There are many types of cancer treatment. Most people have a combination of treatments, such as surgery with chemotherapy and/or radiation therapy. But chemotherapy causes some side effects by damaging healthy cells. They include anaemia, hair loss, nausea and vomiting, appetite changes, constipation, diarrhea, mouth, tongue and throat problems, weight changes etc. Chemotherapy induced nausea and vomiting is a common side effect of many cancer treatments. Modern antiemetics are used to control chemotherapy-induced nausea and vomiting but nausea and vomiting still affects up to $60 \%$ and $37 \%$ of patients undergoing chemotherapy respectively (Bloechi Daum et al., 2006).

Traditionally many Siddha Sashtric formulations have been used for centuries to treat gastrointestinal complaints. They have many therapeutic actions including digestive, stimulant, antioxidant, antiinflammatory, chemo preventive, bioenhancer, anti cancer, immuno modulatory, gastro protective, anti mutagenic, anti ulcer, anti secretory, anti tumerogenic, anti emetic, anti apoptotic, anti hyperglycemic, anti lipidemic, radio protective, hepato protective, anti microbial, anti bacterial, 
Vigneshwari S et.al. New scientific validation on the effect of siddha sashtric formulations in chemotherapy induced nausea and vomiting - a review.

carminative, cytotoxic, anaesthetic, anti cholinesterase activity. So I decided to review the scientific evidence for the effect of Siddha sashtric formulations in the treatment and management of chemotherapy-induced nausea and vomiting.

Information on antiemetic siddha therapeutic formulations was collected from ancient siddha books held in the library, Government siddha medical college, Palayamkottai, Tirunelveli. Electronic searches also made. Information on the chemical constituents and therapeutic actions of the ingredients were collected. This study will be useful for researchers and give relief to the patients undergoing chemotherapy.

Therapeutic formulations used for nausea and vomiting are listed in the table 1. They are available in the forms of medicinal powder, lozenges, electuary, medicated ghee, syrup, distilled extracts.

Table 1: List of medicinal formulations used for nausea and vomiting

\begin{tabular}{|c|c|c|c|c|}
\hline S.NO & $\begin{array}{l}\text { MEDICINAL } \\
\text { FORMULATIONS }\end{array}$ & $\begin{array}{l}\text { PHARMACOLOGICAL } \\
\text { FORM }\end{array}$ & INDICATIONS & REFERENCES \\
\hline 1 & Seeraga chooranam & Medicinal powder & Vomiting, indigestion & Siddha vaidhya thirattu \\
\hline 2 & Inji chooranam - I & Medicinal powder & Vomiting, indigestion & Siddha vaidhya thirattu \\
\hline 3 & Inji chooranam -II & Medicinal powder & Abhorrence of food, vomiting & Siddha vaidhya thirattu \\
\hline 4 & Thaalisaathy vadagam & Lozenges & Vomiting, indigestion & Siddha vaidhya thirattu \\
\hline 5 & Panja deebaakkini ilagam & Electuary & $\begin{array}{ll}\text { Vomiting, } & \text { flatulence, } \\
\text { indigestion } & \end{array}$ & Siddha vaidhya thirattu \\
\hline 6 & Inji ilagam & Electuary & $\begin{array}{l}\text { Abhorrence of food, vomiting, } \\
\text { flatulence }\end{array}$ & Siddha vaidhya thirattu \\
\hline 7 & Puliyaarai nei & Medicated ghee & Tastelessness, vomiting & Siddha vaidhya thirattu \\
\hline 8 & Maadhulai manapagu & Syrup & $\begin{array}{l}\text { Anaemia, tastelessness, } \\
\text { vomiting }\end{array}$ & Siddha vaidhya thirattu \\
\hline 9 & Ealathy chooranam & Medicinal powder & Heartburn, vomiting & $\begin{array}{lll}\begin{array}{l}\text { Marunthu } \\
\text { kalaiyum }\end{array} & \text { sei } & \text { iyalum } \\
\end{array}$ \\
\hline 10 & Kesari ilagam & Electuary & $\begin{array}{l}\text { Heartburn, tastelessness, } \\
\text { nausea, vomiting }\end{array}$ & $\begin{array}{lll}\begin{array}{l}\text { Marunthu } \\
\text { kalaiyum }\end{array} & \text { sei } & \text { iyalum } \\
\end{array}$ \\
\hline 11 & Vilvathy ilagam & Electuary & $\begin{array}{l}\text { Loss of appetite, heartburn , } \\
\text { vomiting }\end{array}$ & $\begin{array}{lll}\begin{array}{l}\text { Marunthu } \\
\text { kalaiyum }\end{array} & \text { sei } & \text { iyalum } \\
\end{array}$ \\
\hline 12 & Ooma theeneer & Distilled extracts & Vomiting, apepsia & $\begin{array}{l}\begin{array}{l}\text { Marunthu } \\
\text { kalaiyum }\end{array} \\
\text { sei }\end{array}$ \\
\hline 13 & Paruththi vithai chooranam & Medicinal powder & Vomiting & $\begin{array}{l}\text { The siddha formulary of } \\
\text { india part }-I I\end{array}$ \\
\hline 14 & Inji manapagu & Syrup & Vomiting, abhorrence of food & Sikitcha rathna deebam \\
\hline
\end{tabular}

\section{PHARMACOLOGICAL FORMS}
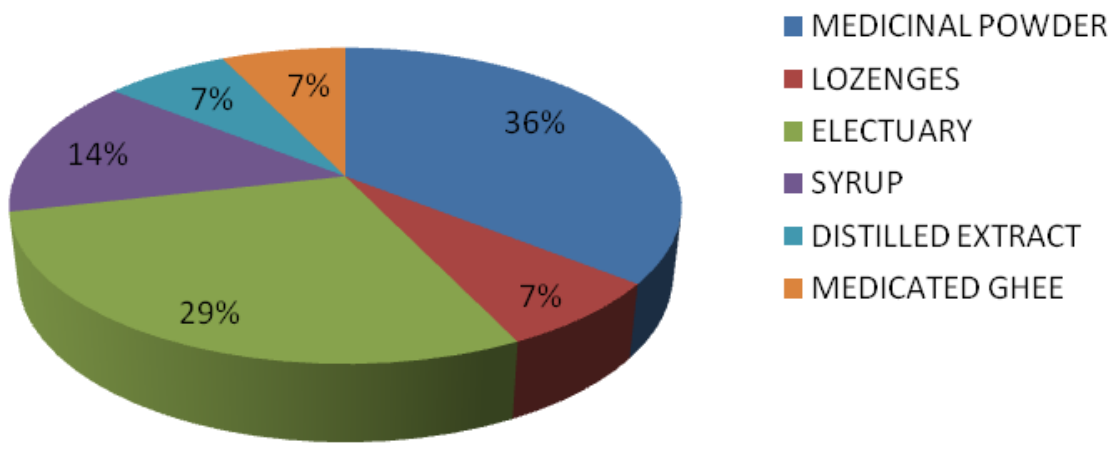

Figure 1: Pharmacological forms of Siddha Antiemetics

Main ingredients of 14 antiemetic therapeutic formulations are given in the table 2. Phytopharmacology of these ingredients are given in the table 3. 
Vigneshwari S et.al. New scientific validation on the effect of siddha sashtric formulations in chemotherapy induced nausea and vomiting - a review.

Table 2: Main ingredients of antiemetic siddha therapeutic formulations

\begin{tabular}{|c|c|c|}
\hline S.NO & $\begin{array}{l}\text { MEDICINAL } \\
\text { FORMULATIONS }\end{array}$ & MAIN INGREDIENTS \\
\hline 1 & Seeraga chooranam & $\begin{array}{l}\text { Cuminum cyminum, Citrus limon, Saccharum officinarum, Mukia madaraspatana, Phyllanthus } \\
\text { emblica, Solanum trilobatum, Azadiracta indica, Leucas aspera. }\end{array}$ \\
\hline 2 & Inji chooranam -I & Zingiber officinale, Cuminum cyminum. \\
\hline 3 & Inji chooranam -II & $\begin{array}{l}\text { Zingiber officinale - fresh and dried rhizome, Piper longum fruit \& root, Piper nigrum, Elattaria } \\
\text { cardamomum, Myristica fragrance pod \& aril, Nardostachys jatamansi, Cuminum cyminum, } \\
\text { Pimpinella anisum, Costus speciosus, Taxus buccata. }\end{array}$ \\
\hline 4 & Thaalisaathy vadagam & $\begin{array}{l}\text { Taxus buccata, Piper longum fruit \& root, Piper nigrum fruit \& root, Zingiber officinale, } \\
\text { Cinnamomum verum, Cinnamomum tamala, Mesua ferrea, Elattaria cardamomum, Vetiveria } \\
\text { zizanoids, alpinia galanga. }\end{array}$ \\
\hline 5 & Panja deebaakkini ilagam & Zingiber officinale, Piper nigrum, Piper longum, Elattaria cardamomum, Cuminum cyminum. \\
\hline 6 & Inji ilagam & $\begin{array}{l}\text { Zingiber officinale - fresh and dried rhizome, Solanum surrattense, Tribulus terrestris, Raphanus } \\
\text { sativus, Citrus limon, Piper nigrum, Piper longum, Elattaria cardamomum, Cuminum cyminum, } \\
\text { Embelia ribs, Syzygium aromaticum, Taxus baccata. }\end{array}$ \\
\hline 7 & Puliya & $\begin{array}{l}\text { Oxalis corniculata, Prosopis cineraria, Alternanthera sesssilis, tender coconut water, Cuminum } \\
\text { cyminum, Elattaria cardamomum, Glycyrrhiza glabra, Quercus infectoria, Myristica fragrans. }\end{array}$ \\
\hline 8 & Maadhulai manapagu & Punica granatum. \\
\hline 9 & Ealathy chooranam & $\begin{array}{l}\text { Elattaria cardamomum, Zingiber officinale, Curcuma angustifolia, Taxus baccata, Mesua ferrea, } \\
\text { Piper nigrum, Syzygium aromaticum. }\end{array}$ \\
\hline 10 & Kesal & $\begin{array}{l}\text { Coriandrum sativum, Piper nigrum, Piper longum, Myristica fragrans, Taxus baccata, Costus } \\
\text { speciosus, Citrus limon. }\end{array}$ \\
\hline 11 & Vilvathy ilagam & $\begin{array}{l}\text { Aegle marmelos, Zingiber officinale, Piper longum, Piper longum, Elattaria cardamomum, } \\
\text { Cinnamomum verum, Mesua ferrea, Syzygium aromaticum, Myristica fragrans, Taxus baccata. }\end{array}$ \\
\hline 12 & Ooma theeneer & Carum copticum. \\
\hline 13 & $\begin{array}{l}\text { Paruththi } \\
\text { chooranam }\end{array}$ & Gossypium arboreum, Limonia acidissima, Oryza sativa, Piper longum, Carum copticum. \\
\hline 14 & Inji manapagu & \\
\hline
\end{tabular}

Table 3: Phytopharmacology of Main Ingredients

\begin{tabular}{|c|c|c|c|c|}
\hline S.NO & INGREDIENTS & $\begin{array}{l}\text { PART } \\
\text { USED }\end{array}$ & CHEMICAL CONSTITUENTS & THERAPEUTIC ACTIONS \\
\hline 1 & Cuminum cyminum $^{1,2}$ & Seed & $\begin{array}{l}\text { Cuminaldehyde, cuminol, cymene, cuminoside, } \\
\text { cimonene, eugenol }\end{array}$ & $\begin{array}{ll}> & \text { Digestive } \\
> & \text { Stimulant } \\
> & \text { Antioxidant } \\
> & \text { Anti inflammatory } \\
> & \text { Chemo preventive } \\
> & \text { Bio enhancer activity }\end{array}$ \\
\hline 2 & Citrus limon $^{3}$ & Fruit & Coumarins, polyphenols, terpenes, citric acid & $\begin{array}{ll}> & \text { Antioxidant } \\
> & \text { Chemo preventive }\end{array}$ \\
\hline 3 & Saccharum officinarum $^{9}$ & Stem & $\begin{array}{l}\text { Phytosterols, flavonoids, glycosides, phenolic acid, } \\
\text { fatty acids }\end{array}$ & $>$ Antioxidant \\
\hline 4 & Mukia maderaspatana $^{4}$ & Leaf & $\begin{array}{l}\text { Phenolic compounds, saponarin, quercetin, } \\
\text { phloroglucinol, flavonoids, steroids, tannin }\end{array}$ & $\begin{array}{l}>\text { Immunomodulatory } \\
>\quad \text { Antioxidant }\end{array}$ \\
\hline 5 & Phyllanthus emblica $^{5}$ & $\begin{array}{l}\text { Unriped } \\
\text { fruit }\end{array}$ & $\begin{array}{l}\text { Emblicanin A \& B, punigluconin, kaempherol, } \\
\text { chebulinic acid, gallic acid }\end{array}$ & $\begin{array}{ll}> & \text { Antioxidant } \\
> & \text { Gastro protective } \\
> & \text { Chemo preventive } \\
> & \text { Anti inflammatory } \\
> & \text { Anti }- \text { mutagenic effect }\end{array}$ \\
\hline 6 & Solanum trilobatum ${ }^{6}$ & Leaf & Saponins, carbohydrates, phytosterols, tannins & $>$ Antioxidant \\
\hline 7 & Azadiracta indica? & Bark & $\begin{array}{l}\text { Azadiractin, nimbolinin, nimbidol, isomeldenin, } \\
\text { nimbin, nimbinene, nimocenol, margocin, } \\
\text { nimbidin, nimban, fnimbosterol, quercetin }\end{array}$ & $\begin{array}{ll} & \text { Anti ulcer } \\
> & \text { Anti secretory } \\
> & \text { Anti cancer } \\
> & \text { Antioxidant } \\
> & \text { Immunomodulatory }\end{array}$ \\
\hline 8 & Leucas aspera $^{8}$ & Leaf & $\begin{array}{l}\text { Triterpene, diterpene, fatty acid ester, fatty acid } \\
\text { amide }\end{array}$ & $\begin{array}{l}>\text { Anti inflammatory } \\
>\quad \text { Anti cancer } \\
\text { Antioxidant }\end{array}$ \\
\hline 9 & Zingiber officinale $^{10}$ & Rhizome & $\begin{array}{l}\text { Gingerol, paradol, shagoal, polyphenols, carotene, } \\
\text { tannins, flavonoids, }\end{array}$ & $\begin{array}{ll}> & \text { Anti inflammatory } \\
> & \text { Anti tumerogenic } \\
> & \text { Immunomodulatory } \\
> & \text { Anti emetic } \\
> & \text { Antioxidant } \\
> & \text { Anti apoptotic } \\
> & \text { Anti hyperglycaemic } \\
> & \text { Anti lipidemic }\end{array}$ \\
\hline 10 & Piper longum $^{11}$ & Fruit & $\begin{array}{l}\text { Piperine, piperlongumine, sylvatin, sesamin, } \\
\text { pipermonaline, piperundecalidine }\end{array}$ & $\begin{array}{ll}> & \text { Carminative } \\
> & \text { Immunomodulatory } \\
> & \text { Antioxidant } \\
> & \text { Anticancer } \\
> & \text { Anti inflammatory } \\
> & \text { Hepatoprotective } \\
> & \text { Anti ulcer }\end{array}$ \\
\hline
\end{tabular}


Vigneshwari S et.al. New scientific validation on the effect of siddha sashtric formulations in chemotherapy induced nausea and vomiting - a review.

\begin{tabular}{|c|c|c|c|c|}
\hline \multicolumn{5}{|c|}{ Table 3: Continued... } \\
\hline 11 & Piper nigrum $^{12}$ & Fruit & $\begin{array}{l}\text { Piperine, piperidine, chavicine, piperetine, } \\
\text { phenolic amino acids, feruperine }\end{array}$ & $\begin{array}{ll}> & \text { Immunomodulatory } \\
> & \text { Antioxidant } \\
> & \text { Anticancer } \\
> & \text { Anti inflammatory } \\
> & \text { Hepatoprotective }\end{array}$ \\
\hline 12 & Piper longum ${ }^{13}$ & Root & $\begin{array}{l}\text { Piperine, piperlongumine, sylvatin, sesamin, } \\
\text { pipermonaline, piperundecalidine }\end{array}$ & $\begin{array}{ll}> & \text { Antibacterial } \\
> & \text { Antidiabetic } \\
> & \text { Anti hyperlipidemic }\end{array}$ \\
\hline 13 & Elattaria cardamomum $^{14}$ & Fruit & $\begin{array}{l}\text { Cineole, limonene, terpinolene, myrcene, } \\
\text { linalool, alpha terpinyl acetate }\end{array}$ & $\begin{array}{ll}> & \text { Chemo preventive } \\
> & \text { Immunomodulatory } \\
> & \text { Anticancer }\end{array}$ \\
\hline 14 & Zingiber officinale ${ }^{10}$ & $\begin{array}{l}\text { Dried } \\
\text { rhizome }\end{array}$ & $\begin{array}{l}\text { Gingerol, paradol, shagoal, polyphenols, } \\
\text { carotene, tannins, flavonoids, }\end{array}$ & $\begin{array}{ll}> & \text { Anti inflammatory } \\
> & \text { Anti tumerogenic } \\
> & \text { Immunomodulatory } \\
> & \text { Anti emetic } \\
> & \text { Antioxidant } \\
> & \text { Anti apoptotic } \\
> & \text { Anti hyperglycaemic } \\
> & \text { Anti lipidemic }\end{array}$ \\
\hline 15 & Myristica fragrans $^{15}$ & Pod & $\begin{array}{l}\text { Myristicin, myristic acid, elemicin, safrole, } \\
\text { trimyrishtin, myrcene }\end{array}$ & $\begin{array}{ll}> & \text { Anti inflammatory } \\
> & \text { Immunomodulatory } \\
> & \text { Antioxidant } \\
> & \text { Carminative } \\
> & \text { Hepatoprotective } \\
> & \text { Stimulant } \\
> & \text { Radio protective }\end{array}$ \\
\hline 16 & Nardostachys jatamansi $^{16}$ & Root & $\begin{array}{l}\beta \text { - eudesmol, elemol, } \beta \text {-sitisterol, algelicin, } \\
\text { jatamansin }\end{array}$ & $\begin{array}{ll}> & \text { Anti cancer } \\
> & \text { Antioxidant } \\
> & \text { Hepatoprotective }\end{array}$ \\
\hline 17 & Costus speciosus $^{18}$ & Root & $\begin{array}{l}\text { Tigogenin, diosgenin, } \\
\text { cycloartenol, octacosanoic acid }\end{array}$ diosgenone, & $\begin{array}{ll}> & \text { Antioxidant } \\
> & \text { Anti inflammatory } \\
> & \text { Anti cancer } \\
> & \text { Hepatoprotective } \\
> & \text { Anti cholinesterase }\end{array}$ \\
\hline 18 & Myristica fragrans ${ }^{20}$ & Aril & $\begin{array}{l}\text { Myristicin, myristic acid, elemicin, safrole, } \\
\text { trimyrishtin, myrcene }\end{array}$ & $\begin{array}{ll}> & \text { Anti inflammatory } \\
> & \text { Immunomodulatory } \\
> & \text { Antioxidant } \\
> & \text { Radio protective } \\
\end{array}$ \\
\hline 19 & Taxus baccata $^{19}$ & Leaf & Taxol, taxane, taxine, taxoids & $>\quad$ Anti cancer \\
\hline 20 & Piper nigrum $^{12}$ & Root & $\begin{array}{l}\text { Piperine, piperone, pipertol, nerol, hexenol, } \alpha \\
\text { - terpinol,acetophenone }\end{array}$ & $\begin{array}{ll}> & \text { Antioxidant } \\
> & \text { Anticancer } \\
> & \text { Anti inflammatory }\end{array}$ \\
\hline 21 & Cinnamomum verum $^{23}$ & Bark & $\begin{array}{l}\text { Eugenol, } \begin{array}{l}\text { E- cinnamyl } \\
\text { cinnamaldehyde, caryophyllene oxide }\end{array} \\
\text { acetate, }\end{array}$ & $\begin{array}{ll}> & \text { Anti microbial } \\
> & \text { Antioxidant } \\
> & \text { Anticancer } \\
> & \text { Anti inflammatory } \\
> & \text { Anti diabetic } \\
\end{array}$ \\
\hline 22 & Cinnamomum tamala ${ }^{24}$ & Leaf & $\begin{array}{l}\text { Pinene, myrcene, camphene, p- cymene, } \\
\text { limonene, eugenol, methyl eugenol }\end{array}$ & $\begin{array}{ll}> & \text { Antioxidant } \\
> & \text { Immuno suppressive } \\
> & \text { Carminative } \\
> & \text { Gastro protective }\end{array}$ \\
\hline 23 & Mesua ferrea $^{25}$ & Flower & $\begin{array}{l}\alpha-\text { copaene, biflavonoids, rhusflavanone, } \\
\text { mesuaferrone B }\end{array}$ & $\begin{array}{ll}> & \text { Anti microbial } \\
> & \text { Immuno modulator } \\
> & \text { Antioxidant } \\
> & \text { Anti inflammatory } \\
> & \text { Hepato protective }\end{array}$ \\
\hline 24 & Elattaria cardamomum ${ }^{14}$ & Seed & $\begin{array}{l}\text { Cineole, limonene, terpinolene, myrcene, } \\
\text { linalool, alpha terpinyl acetate }\end{array}$ & $\begin{array}{l}>\text { Chemo preventive } \\
>\quad \text { Immunomodulatory } \\
>\quad \text { Anticancer }\end{array}$ \\
\hline 25 & Vetiveria zizanioids $^{26}$ & Root & $\begin{array}{l}\beta-\text { vetivenene, } \beta \text {-vetivone, } \alpha \text { - vetivone, } \\
\text { vetisenlinenol, } \beta \text { - vetispirene, khusimol }\end{array}$ & $\begin{array}{ll}> & \text { Antioxidant } \\
> & \text { Cytotoxic } \\
> & \text { Anti tuberculosis }\end{array}$ \\
\hline 26 & Alpinia galanga $^{27}$ & Rhizome & $\begin{array}{l}\text { Alpinin, galangol, galangin, campheride, } \\
\text { galanolactone }\end{array}$ & $\begin{array}{ll}> & \text { Immuno stimulating activity } \\
> & \text { Antioxidant } \\
> & \text { Anti inflammatory } \\
> & \text { Anticancer } \\
> & \text { Anti ulcer } \\
> & \text { Carminative } \\
> & \text { Gastro protective }\end{array}$ \\
\hline 27 & Solanum surrattense $\mathrm{e}^{28}$ & Leaf & $\begin{array}{l}\text { Solasonine, } \\
\text { diosgenin }\end{array}$ campestrol, $\quad$ campeferol, & $\begin{array}{ll}> & \text { Hepatoprotective } \\
> & \text { Antioxidant } \\
> & \text { Anti apoptotic } \\
> & \text { Anti bacterial } \\
\end{array}$ \\
\hline
\end{tabular}


Vigneshwari S et.al. New scientific validation on the effect of siddha sashtric formulations in chemotherapy induced nausea and vomiting - a review.

\begin{tabular}{|c|c|c|c|c|}
\hline \multicolumn{5}{|c|}{ Table 3: Continued... } \\
\hline 28 & Tribulus terrestris $^{29}$ & $\begin{array}{l}\text { Whole } \\
\text { plant }\end{array}$ & $\begin{array}{l}\text { Terrestrin C,D,E, kaempherol, tribulusterine, } \\
\text { vanillin, furostanol }\end{array}$ & $\begin{array}{ll}> & \text { Antioxidant } \\
> & \text { Immunomodulatory } \\
> & \text { Anticancer } \\
> & \text { Anti inflammatory } \\
> & \text { Hepatoprotective } \\
> & \text { Absorption enhancing activity }\end{array}$ \\
\hline 29 & Raphanus sativus $^{30}$ & Root tuber & Glucosinolate, myrosinase, isothiocyanate & $\begin{array}{ll}> & \text { Chemo preventive } \\
> & \text { Cytotoxic } \\
> & \text { Anti microbial }\end{array}$ \\
\hline 30 & Embelia ribes $^{31}$ & Fruit & $\begin{array}{l}\text { Embelin, embolic acid, embelinol, } \\
\text { christembine, cinnamic acid, rapanon }\end{array}$ & $\begin{array}{ll}> & \text { Antioxidant } \\
& \text { Cardio protective }\end{array}$ \\
\hline 31 & Syzygium aromaticum ${ }^{22}$ & Bud & $\begin{array}{l}\text { Myricetin, eugenol, eugenol acetate, limonin, } \\
\text { ferulic aldehyde }\end{array}$ & $\begin{array}{ll}> & \text { Anti cancer } \\
> & \text { Anaesthetic } \\
> & \text { Antioxidant } \\
> & \text { Anti inflammatory } \\
> & \text { Anti microbial }\end{array}$ \\
\hline 32 & Oxalis corniculata $^{32}$ & Leaf & Galacto - glycerolipid, isutiexin, volatile oil & $\begin{array}{ll}> & \text { Antioxidant } \\
> & \text { Anti inflammatory } \\
> & \text { Anti microbial } \\
> & \text { Anti cancer } \\
> & \text { Anti ulcer } \\
> & \text { Hepato protective }\end{array}$ \\
\hline 33 & Prosopis cineraria ${ }^{35}$ & Leaf & 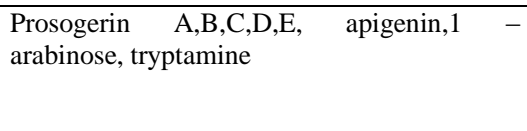 & $\begin{array}{ll}> & \text { Anti cancer } \\
> & \text { Antioxidant } \\
> & \text { Anti inflammatory } \\
> & \text { Anti microbial }\end{array}$ \\
\hline 34 & Alternanthera sessilis s3 $^{33}$ & Leaf & $\begin{array}{l}\beta-\text { carotene, } \alpha-\text { spirasterol, uronic acid, } \beta- \\
\text { sitosterol, ricinoleic acid, myristic, palmitic, } \\
\text { stearic, oleic and linoleic acids }\end{array}$ & $\begin{array}{ll}> & \text { Anti microbial } \\
> & \text { Anti cancer } \\
> & \text { Hepato protective }\end{array}$ \\
\hline 35 & Tender coconut water $^{34}$ & - & Auxin, gibberellins & $\begin{array}{ll}> & \text { Hepato protective } \\
> & \text { Antioxidant } \\
> & \text { Anti inflammatory }\end{array}$ \\
\hline 36 & Glycyrrhiza glabra $^{37}$ & Root & $\begin{array}{l}\text { Glycyrrhizin, } \quad \text { glabrin, liqurtin, } \\
\text { semilicoisoflavone, methoxyficifolinol }\end{array}$ & $\begin{array}{ll}> & \text { Anti inflammatory } \\
> & \text { Anti ulcer } \\
> & \text { Antioxidant } \\
> & \text { Anti cancer } \\
> & \text { Anti hepato toxic }\end{array}$ \\
\hline 37 & Quercus infectoria $^{36}$ & $\begin{array}{l}\text { Unriped } \\
\text { fruit }\end{array}$ & $\begin{array}{l}\text { Tannin, gallic acid, ellagic acid, pyrogallol, } \\
\text { syringic acid, amentoflavone }\end{array}$ & $\begin{array}{ll}> & \text { Antioxidant } \\
> & \text { Anti inflammatory } \\
> & \text { Cytotoxic } \\
> & \text { Anti leismanial }\end{array}$ \\
\hline 38 & Punica granatum ${ }^{21}$ & Fruit & $\begin{array}{l}\text { Punicalagin, oleanic acid, ellagitanins, } \\
\text { gallotanic acid, anthocyanins, phenols, } \\
\text { flavonoids }\end{array}$ & $\begin{array}{ll}> & \text { Chemo preventive } \\
> & \text { Antioxidant } \\
> & \text { Anti inflammatory } \\
> & \text { Anti carcinogenic } \\
\end{array}$ \\
\hline 39 & Curcuma angustifolia $^{41}$ & Root tuber & Curzerenone, camphor, germacrone & $>$ Antioxidant \\
\hline 40 & Coriandrum sativum $^{38}$ & Seed & $\begin{array}{l}\text { Linalool, decanoic acid, capric acid, nerol,cis } \\
\text { - dihydrocarvone, decanal }\end{array}$ & $\begin{array}{ll}> & \text { Antioxidant } \\
> & \text { Anti inflammatory } \\
> & \text { Hepatoprotective }\end{array}$ \\
\hline 41 & Aegle marmelos ${ }^{48}$ & Root & $\begin{array}{l}\text { Marmelosine, aeglemarmelosine, marmine, } \\
\text { marmisine }\end{array}$ & $\begin{array}{ll}> & \text { Antioxidant } \\
> & \text { Chemo preventive } \\
> & \text { Anti inflammatory } \\
> & \text { Anti cancer } \\
> & \text { Ulcer healing activity }\end{array}$ \\
\hline 42 & Carum copticum ${ }^{39}$ & Seed & $\begin{array}{l}\text { Paracymene, } \beta \text { - pinene,thymol, terpinolene, } \\
\text { o- cymene, carracol }\end{array}$ & $\begin{array}{ll}> & \text { Antioxidant } \\
> & \text { Carminative } \\
> & \text { Hepatoprotective } \\
> & \text { Anti inflammatory } \\
> & \text { Anti emetic } \\
> & \text { Anti mutagenic activity }\end{array}$ \\
\hline 43 & Gossypium arboreum $^{49}$ & Seed & $\begin{array}{l}\text { Gossypol, cyclopropenoid, linoleic acid, } \\
\text { oleic acid, palmitic acid }\end{array}$ & $\begin{array}{ll}> & \text { Antioxidant } \\
>\quad \text { Anti bacterial }\end{array}$ \\
\hline 44 & Limonia acidissima $^{40}$ & Seed & $\begin{array}{ll}\text { Maemesin, } & \text { marmin, feronia lactone, } \\
\text { feronone, } & \text { umbelliferone, dictamnine, } \\
\text { scroparone } & \end{array}$ & $\begin{array}{ll}> & \text { Antioxidant } \\
> & \text { Anti microbial }\end{array}$ \\
\hline 45 & Oryza sativa $^{50}$ & Seed & $\begin{array}{l}\text { Starch, glucose, fructose, galactose, glutamic } \\
\text { acid, aspartic acid }\end{array}$ & $>$ Antioxidant \\
\hline 46 & Foeniculum vulgare $^{17}$ & Seed & $\begin{array}{l}\text { Alpha pinene, beta myrcene, beta pinene, } \\
\text { bitter fenchone, camphene, estragole, } \\
\text { fenchone, limonene, p-cymen, safrole. }\end{array}$ & $\begin{array}{ll}> & \text { Antioxidant } \\
> & \text { Anti inflammatory } \\
> & \text { Anticancer } \\
> & \text { Hepato protective } \\
> & \text { Carminative } \\
> & \text { Digestive }\end{array}$ \\
\hline
\end{tabular}




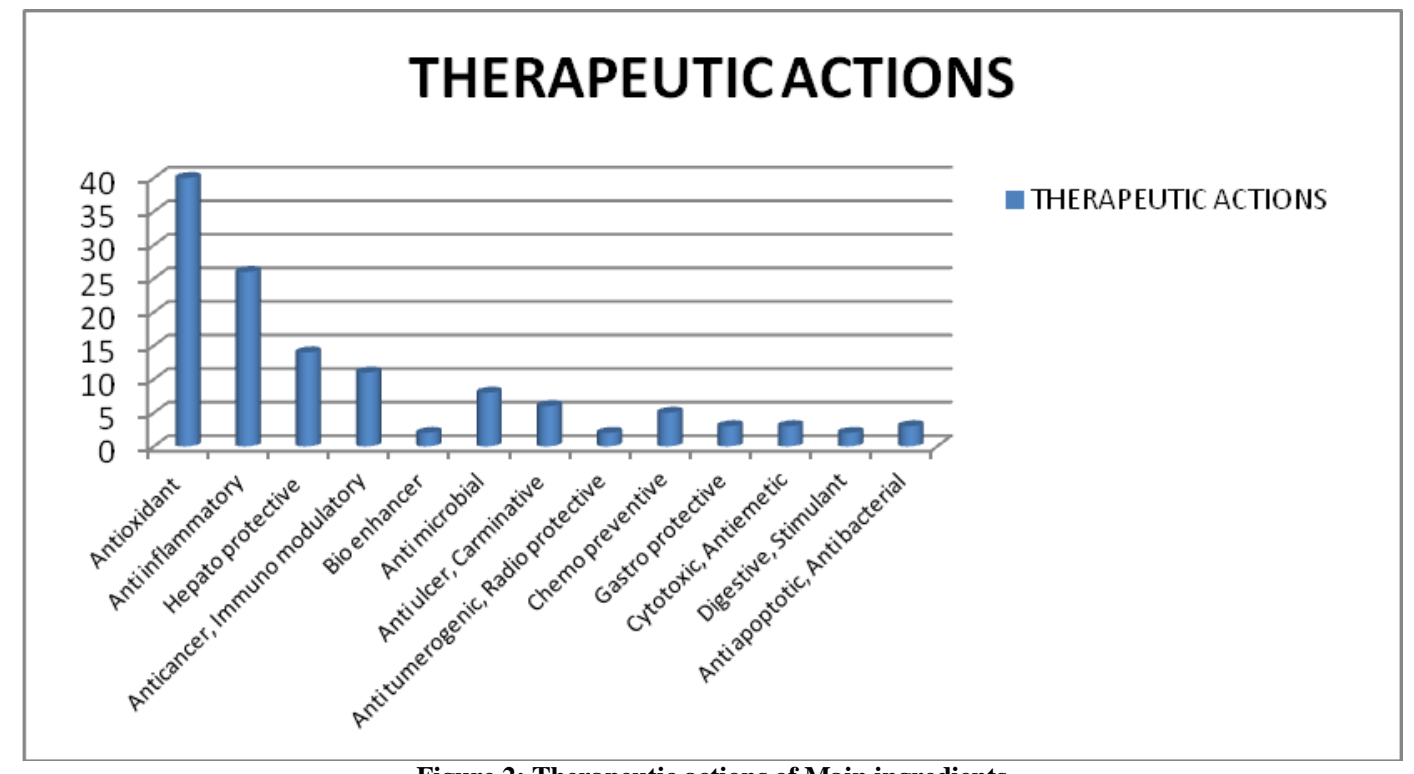

Figure 2: Therapeutic actions of Main ingredients

Totally 46 ingredients are reviewed. They have digestive (2), stimulant (2), antioxidant (40), anti-inflammatory (26), chemo preventive (5), bio-enhancer (2), anti cancer (11), immuno modulatory (11), gastro protective (3), anti mutagenic (1), anti ulcer (6), anti secretory (1), anti tumerogenic (2), anti emetic (3), anti apoptotic (3), anti hyperglycaemic (1), anti lipidemic (2), radio protective (2), hepato protective (14), anti microbial (8), anti bacterial (3), carminative (6), cytotoxic (3), anaesthetic (1), anti cholinesterase (1), cardio protective (1), anti tuberculosis (1), anti leishmanial (1) activities. So these formulations definitely cure and manage CINV and reduce the burden of patients undergoing chemotherapy.

\section{CONCLUSION}

Considering the therapeutic actions and chemical constituents of the ingredients in Siddha antiemetic therapeutic formulations, a need was felt to carry out further preclinical studies and clinical trials on these formulations. This will provide a sound foundation for continuous research in this area. This will be useful for the researchers and the patients undergoing chemotherapy.

\section{Acknowledgement: None}

\section{Conflict of Interest: None}

\section{Source of Funding: None}

\section{REFERENCES}

1. Krishnapura srinivasan. Cumin and black cumin seeds: traditional uses, chemical constituents, and nutraceutical effects. Food quality and safty $2008 ; 2(1): 1-16$.

2. Johri R K. Cuminum cyminum and carum carvi: an update. Pharmacognacy reviews 2011; 5(9): 63-72.

3. Yoshiaki miyake, akira murakami et al,. Identification of coumarins from lemon fruit as inhibitors of in vitro tumor promotion and superoxide and nitric oxide generation. Journal of agricultural and food chemistry 1999; 47(8): 3151-3157.

4. Petrus A J A et al,. Antioxidative constituents of mukia maderaspatana (Linn.) M. Roem. Leaves. IJNPR 2011; 2(1): 34-43.

5. Mirunalini S, Krishnaveni M. Therapeutic potential of Phyllanthus emblica: the ayurvedic wonder. Journal of basic and clinical physiology and pharmacology 2010; 21(1): 93-105.

6. Kumar ganesan, kumeshini sukalingam et al,. Solanum trilobatum L. Ameliorate thioacetamide - induced oxidative stress and hepatic damage in albino rats. Antioxidants 2017; 6, 68 .

7. Uday Bandyopadhyay et al,. Clinical studies on the effect of neem bark extract on gastric secretion and gastroduodenal ulcer. Life sciences 2004; 75(24): 2867-2878. 
8. Prajapati M S et al,. Leucas aspera: a review. Pharmacognosy reviews 2010; 4(7): 85-87.

9. Joaquim Mauricio Duarte - Almeida et al,. Antioxidant activity of phenolic compounds from sugar cane juice. Plant foods for human nutrition 2006; 61: 187-192.

10. Badreldin $\mathrm{H}$ ali, Gerald blunden et al,. Some phytochemical, pharmacological and toxicological properties of ginger: a review of recent research. Food and chemical toxicology 2008; 46(2): 409-420.

11. Vaishali Yadav, Anuja Krishnan et al,. A systematic review on piper longum L.: bridging traditional knowledge and pharmacological evidence for future translational research. Journal of ethnopharmacology 2020; 247,112255.

12. Zoheir A damanhouri, Aftab Ahmad. A review on therapeutic potential of Piper nigrum L. The king of spices 2014; 3, 161.

13. Shaik abdul nabi, ramesh babu kasetti et al,. Antidiabetic and antihuperlipidemic activity of piper longum root aqueous extract in STZ induced diabetic rats. BMC complementary and alternative medicine 2013; 13(1): 37.

14. Samir qiblawi, Awdah Al - Hazimi et al,. Chemopreventive effects of Cardamom on chemically induced skin carcinogenesis in swiss albino mice. Journal of medicinal food 2012; 15(6): 576-580.

15. Rahul checker, Suchandra chatterjee et al,. Immunomodulatory and radioprotective effect of lignans derived from fresh nutmeg mace in mammalian splenocytes. International immunopharmacology 2008; 8(5): 661-669.

16. Shilpee chaudhary, Kodangala Subraya et al,. Evaluation of antioxidant and anticancer activity of extract and fractions of Nardostachys jatamansi DC in breast carcinoma. BMC complementary and alternative medicine 2015; 15(1): 1-13.

17. Wesam Kooti, Maryam Moradi et al,. Therapeutic and pharmacological potential of Foeniculum vulgare Mill: a review. Journal of HerbMed pharmacology 2015; 4(1): 1-9.

18. Ali hafez el-far et al,. Costus speciosus: traditional uses, phytochemistry, and therapeutic potentials. Pharmacognosy reviews 2018; 12(23).

19. Abolghesem abbasi kajani et al,. Anticancer effects of silver nanoparticles encapsulated by Taxus baccata extracts. Journal of molecular liquids 2016; 223: 549-556.

20. Yukihiro ozaki, Soekeni Soedigdo et al,. Anti-inflammatory effect of mace, aril of myristica fragrans Houtt., and its active principles. The Japanese journal of pharmacology 1989; 49(2): 155-163.

21. Nam Deuk Kim, Rajendra Mehta et al,. Chemopreventive and adjuvant therapeutic potential of pomegranate for human breast cancer. Breast cancer research and treatment 2002; 71(3): 203-207.

22. Arunava Das, Harshadha $\mathrm{K}$ et al,. Evaluation of therapeutic potential of eugenol - a natural derivative of syzygium aromaticum on cervical cancer. Asian pacific journal of cancer prevention 2018; 19(7): 1977.

23. Neetu singh, Amerender Singh rao et al,. Phytochemical and pharmacological review of Cinnamomum verum J. Presl - a versatile spice used in food and nutrition. Food chemistry 2020; 338, 127773.

24. Bavani eswaran M, Surendran $S$ et al,. Gastroprotective activity of Cinnamomum tamala leaves on experimental gastric ulcers in rats. Journal of ethnopharmacology 2010; 128(2): 537-540.

25. Manoj kumar chahar, sanjaya kumarDS et al,. Mesua ferrea L: a review of the medical evidence for its phytochemistry and pharmacological actions. African journal of pharmacy and pharmacology 2013; 7(6): 211-219.

26. Ritu Gupta et al,. Anticonvulsant activity of ethanol extracts of Vetiveria zizanioides roots in experimental mice. Pharmaceutical biology 2013; 51(12): 1521-1524.

27. Chudiwal AK, jain DP et al,. Alpinia galanga Willd. - an overview on phyto pharmacological properties. CSIR 2010.

28. Sridevi Muruhan, Senthil Selvaraj et al,. In vitro antioxidant activities of Solanum surrattense leaf extract. Asian pacific journal of tropical biomedicine 2013; 3(1): 28-34.

29. Sri ranjani sivapalan. Biological and pharmacological studies of Tribulus terrestris Linn - a review. Int $\mathbf{J}$ multidiscip res dev 2016; 3(1): 257-265.

30. Mohemed A abd - elmoneim et al,. Anticarcinogenic effect of Raphanus sativus on 1, 2 dimethylhydrazine (DMH) induced colon cancer in rats. The Egyptian journal of hospital medicine 2013; 51(1): 473-486. 
31. Uma bhandari, Nazam Ansari $M$ et al,. Cardioprotective effect of aqueous extract of Embelia ribes Burm fruits against isoproterenol - induced myocardial infarction in albino rats. Csir 2008.

32. Kathiriya A, Das K et al,. Evaluation of antitumor and antioxidant activity of oxalis corniculata Linn. Against Ehrlich ascites carcinoma on mice. International journal of cancer management 2010; 3(4): 157-165.

33. Sivapragasam gothai et al,. Anticancer potential of Alternanthera sessilis extract on HT-29 human colon cancer cells. Asian pacific journal of tropical biomedicine 2018; 8(8): 394.

34. Shubhashree MN, venkateshwarlu $\mathrm{G}$ et al,. Therapeutic and nutritional values of narikelodaka - a review. Research journal of pharmacognosy and phytochemistry 2014; 6(4): 195-201.

35. Khandelwal preeti, Sharma ram avatar et al,. Pharmacology, phytochemistry and therapeutic application of Prosopis cineraria Linn.: a review. Journal of plant sciences 2015; 3(1-1): 33-39.

36. Gurpreet kaur, Hinna hamid et al,. Antiinflammatory evaluation of alcoholic extract of galls of Quercus infectoria. Journal of ethnopharmacology2004; 90(2-3): 285-292.

37. Rajandeep kaur, Harpreet Kaur et al,. Glycyrrhiza glabra: a phytopharmacological review. International journal of pharmaceutical sciences and research 2013; 4(7): 2470.

38. Jinous asgarpanah et al,. Phytochemistry, pharmacology and medicinal properties of Coriandrum sativum L. African journal of pharmacy and pharmacology 2012; 6(31): 2340-2345.

39. Maryam zahin et al,. Antioxidant and antimutagenic activity of Carum copticum fruit extracts. Toxicology in vitro 2010; 24(4): 1243-1249.

40. Ilango $\mathrm{K}$, chitra V. Antidiabetic and antioxidant activity of Limonia acidissima Linn. In alloxan induced rats. Der pharmacia letter 2009; 1(1): 117-125.
41. Sudipta jena, asit ray et al,. Chemical composition and antioxidant activity of essential oil from leaves and rhizomes of Curcuma angustifolia Roxb. Natural product research 2017; 31(18): 2188-2191.

42. Siddha vaidhya thirattu. Kuppusamy mudhaliyar KN, Uththamarayan KS, Department of Indian medicine and homeopathy, Chennai, $3^{\text {rd }}$ edition, 2009.

43. Marunthu sei iyalum kalaiyum. Dr.Deva asirvatham. Department of Indian medicine and homeopathy, Chennai. 2014.

44. Sikitcharathnadeepam. Kannusami pillai. B. Rathnanayakar \& sons. 1951.

45. Siddha material madica (medicinal plant division). Murugesa mudhaliyar KS. Department of Indian medicine and homeopathy, Chennai. $9^{\text {th }}$ edition, 2013.

46. The Siddha formulary of India part 2. The controller of publications, civil lines, Delhi, $1^{\text {st }}$ edition, 2011.

47. Wolfgang marx et al,. Ginger - mechanism of action in CINV: a review. Critical reviews in food sciences and nutrition 2017; 57(1): 141-146.

48. Shahedur rahman, Rashida parvin. Therapeutic potential of aegle marmelos $\mathrm{L}$. - an overview. Asian pacific journal of tropical diseases 2014. 4(1): 71-77.

49. Kofi annan, peter J Houghton. Antibacterial, antioxidant and fibroblast growth stimulation of aqueous extracts of Ficus asperifolia Miq. And Gossypium arboretum L., wound healing plants of Ghana. Journal of ethnopharmacology 2008; 119(1): 141144.

50. Bruno burlando, laura cornara. Therapeutic properties of rice constituents and derivatives: a review update. Trends in food science \& technology 2014; 40(1): 82-98.

How to cite this article: Vigneshwari S, Antony Duraichi R. New scientific validation on the effect of siddha sashtric formulations in chemotherapy induced nausea and vomiting - a review. Int J Health Sci Res. 2021; 11(4): 182189. DOI: https://doi.org/10.52403/ijhsr. 20210424 